Dr. v. Glo e d e n: Ueber das Kalkbedürfniss der Vögel. 283

Horste). Was hatte nun die Krähe auf dem Bussardhorste zu thun, doch wohl nichts Anderes, als das Ei zu rauben?

In meiner Jugend habe ich beobachtet, wie die Krähen aus einer Reihercolonie im Forstort Dolle in der Letzlinger Haide, R.-B. Magdeburg, die Eier holten, mit diesen auf die Erde flogen und sie dort verzehrten.

Altenkirchen, den 9. Juni 1876.

\title{
Ueber die Quelle, woher die Vögel ihr Kalkbedïrfniss zur Bildung der Eischale befriedigen.
}

Von

Dr. v. Gloeden.

Alle Ornithologen und praktischen Vogelwirthe sind meines Wissens darüber einig, dass die Vögel ihre Eischale aus direct aufgenommenem kohlensaurem Kalke bilden, und dass mithin der gefangene Vogel einer besonderen Kalkfütterung durch Verabreichung von Eierschalen, Kreide oder os sepiae bedürfe; ich glaube dem entschieden widersprechen zu müssen, aus Gründen, die ich in Nachstehendem darzuthun versuchen werde.

Für viele Vögel dürfte zunächst die directe Kalkaufnahme nahezu an die Unmöglichkeit grenzen, wie es z. B. bei der Nachtschwalbe, Thurmsegler, Honigsauger u. s. w. der Fall ist; trotzdem der Kalk nach dem Silicium vielleicht das weitverbreitetste Mineral ist, so kommt er doch nur ausnahmsweise in solcher Form und Menge vor, dass selbst ein Finke ihn absichtlich aufpicken könnte. Man ist wahrscheinlich auf den Gedanken, dass der Vogel einer besonderen Kalkaufnahme bedürfe, durch die Beobachtung gekommen, dass z. B. unsere Hühner und Tauben Kalk an den Wänden picken, wenigstens C. Vogt - Zoologische Briefe, Frankfurt 1851, II, p. 319 - bezieht sich hierauf, vergisst aber bei diesem Benehmen den Mangel des geschlechtlichen Unterschiedes zu erklären, und übersieht überdem, dass man von Culturzuständen keine Schlüsse auf natürliche Verhältnisse wagen darf, denn es unterliegt keinem Zweifel, dass jene Vögel fortfahren würden kalkschalige Eier zu legen, wenn alles Kalkmauerwerk plötzlich von der Erde verschwände und wir unsere Gebäude mit Lehm oder anderen Kitten aufführten. Viele Vögel lieben es zur Beförderung der Verdauung Quarzkörner zu verschlucken; der Mörtel besteht aus Kalk und recht grobem Sande, es ist daher wahrscheinlich, dass 
die Thiere es bei ihrem Benehmen auf die mechanische Wirkung der Kalksandkörnchen abgesehen haben. Manche sind auch Verehrer des Salzgeschmackes, z. B. die Tauben; an feuchten Mauern, besonders in der Nähe von Dunggruben, Stallungen u. s. w., bildet sich in der Regel Salpeter, und es ist daher anzunehmen, dass sie zugleich diesem nachgehen.

Auf der andern Seite behauptet man, reichliche Kalkfütterung helfe über die Legenoth hinweg, und auch Brehm - Gefangene Vögel I, pag. 87, 248 - empfiehlt dieselbe nachdrücklichst; es ist der Grund hierfür indessen nicht recht einzusehen, denn ein weiches oder schalenloses Ei wird sich unbedingt leichter ausscheiden lassen, als ein durch Kalkinkrustirung unplastisch gewordenes. Der Grund der Legenoth ist wohl nur in Körper- und Constitutionsschwächen oder momentanen Störungen zu suchen, wenn nicht ungewöhnliche Grösse und Deformität des Eies die Schuld tragen.

Die Eischale bildet sich bekanntlich erst kurz vor dem Eintritt des Eies in die Kloake durch Secretion im Eileiter; es wird also auch hier der dazu nöthige kohlensaure Kalk vorhanden sein oder fehlen, im letzten Falle werden nur mit der Schalenhaut versehene, sogenannte Windeier zu Tage gefördert. Dass aber ein Mangel an Kalkausscheidung im Eileiter der Vögel auf Mangel an Kalkfutter, wie Brehm - Gef. Vögel I, p. 87 - glaubt, hinweist, ist damit noch keineswegs erwiesen, sondern vielmehr anzunehmen, dass ein solches Vorkommen auf eine zu starke Ausscheidung auf anderem Wege, mithin auch gestörtes Gleichgewicht im Organismus hindeutet, denn so lange der Vogel Kalk durch die Excremente abstösst, ist zuverlässig kein Kalkmangel vorhanden; zunächst befriedigt der gesunde Organismus seine Bedürfnisse, und erst dann wird das Unbrauchbare mit dem Ueberflüssigen entfernt. Die Vermischung der Auswurfstoffe mit Kalk ist aber am allerwenigsten ein Bedürfniss. Auch dürfte eine Frühgeburt bei Vögeln eben so möglich sein, als sie bei Säugethieren thatsächlich vorkommt.

Dagegen enthält sämmtliches Futter, fast ohne Ausnahme, Kalk, manches, wie z. B. der Same von Phalaris canariensis so viel, dass er sogar seines phosphorsauren Kalkes wegen technisch zum Feuchthalten des Geschiebes benutzt wird, und würde derselbe dadurch dem Organismus nicht mehr wie hinreichend zugeführt, so würde derselbe sicher im gesunden Zustande keinen Kalk durch die Excremente ausscheiden; erst wenn dieses Phänomen ausbleibt, ist auf fehlerhafte Fütterung und auf Kalkmangel $\mathrm{zu}$ schliessen. 
Ein Kanarienvogelweibchen, das ich zwei Jahre lang ausschliesslich mit Buchweizen, Grünzeug nach der Jahreszeit fütterte, legte in dieser Zeit 28 vollkommen normalschalige Eier; auch in diesem Fall ist mithin Vogt an der angeführten Stelle im Irrthum. Thatsächlich ist auch z. B., dass der Kanarienvogel, Männchen sowohl als Weibchen, während der Mauser und im Winter mindestens doppelt soviel Eischalen, und zwar gekochte lieber als rohe, aufknabbert als während der Brutzeit.

Die Pflanzenwelt allein ist die Quelle für die Mineralbedürfnisse der ganzen Thierwelt, sie nimmt das aufgelöste Mineral auf und organisirt es, und nur in dieser Form wird es von der Thierwelt weiter verarbeitet. Die pflanzlichen Gebilde des Meeres sind daher die "organischen Filtrirmaschinen" für die Anhäufung des Kalkes, nicht aber die Schalthiere, wie Vogt annimmt - Geologie und Peterefacten-Kunde, Braunschweig 1854 I, pag. 59 - und deshalb ist es auch durchaus nicht „merkwürdig“, wie Jener glaubt, dass die Schalthiere bei dem geringen Kalkgehalte des Meerwassers, das nicht viel über $0,1 \%$ beträgt, ihre mitunter kolossalen Gehäuse bauen, denn die Nahrung, mag sie der Pflanzen- oder Thierwelt angehören, liefert ihnen das Nöthige in bereits concentrirter und organisirter Form. Das ist die Arbeitstheilung im Haushalte der Natur!

Leider muss ich es mir versagen, dieses interessante Thema weiter in's Allgemeine zu verfolgen, denn ich entferne mich zu weit von dem Reiche der Vögel, dem diese Zeilen ausschliesslich gewidmet sein sollten.

\section{Die im Museum zu Lissabon befindlichen Vögel der westafrikanischen Besitzungen Portugals. Von}

Prof. Barboza du Bocage,

übersetzt und zusammengestellt von

Hans Gadow, stud. rer. nat.

In der zweiten Nummer des von der königlichen Akademie der Wissenschaften zu Lissabon 1867 herausgegebenen „Jornal de sciencias mathematicas, physicas e naturaes" brachte Herr Prof. J. V. Barboza du Bocage, Director des dortigen zoolog. Museums, eine Liste der aus den westafrikanischen Besitzungen Portugals erhaltenen Vögel. Bis zum Jahr 1874 erschienen, wie gerade die Sendungen aus Afrika eintrafen, 10 Listen, so dass das darin enthaltene Material als ein schätzenswerther Beitrag der westafr. 


\section{$2 \mathrm{BHL}$ Biodiversity Heritage Library}

Gloeden, V. 1876. "Ueber die Quelle, woher die Vögel ihr Kalkbedürfniss zur Bildung der Eischale befriedigen." Journal fu

r Ornithologie 24, 283-285. https://doi.org/10.1007/bf02004128.

View This Item Online: https://www.biodiversitylibrary.org/item/101769

DOI: https://doi.org/10.1007/bf02004128

Permalink: https://www.biodiversitylibrary.org/partpdf/141484

\section{Holding Institution}

Smithsonian Libraries

\section{Sponsored by}

Biodiversity Heritage Library

\section{Copyright \& Reuse}

Copyright Status: Public domain. The BHL considers that this work is no longer under copyright protection.

This document was created from content at the Biodiversity Heritage Library, the world's largest open access digital library for biodiversity literature and archives. Visit BHL at https://www.biodiversitylibrary.org. 